\title{
High-Resolution Ultrasound Imaging
}

\author{
A Novel Technique for the Noninvasive in Vivo Analysis of \\ Endometriotic Lesion and Cyst Formation in Small Animal \\ Models
}

Matthias W. Laschke, ${ }^{*}$ Christina Körbel, ${ }^{*}$ Jeannette Rudzitis-Auth, ${ }^{*}$ Isabella Gashaw, ${ }^{\dagger}$ Michael Reinhardt, ${ }^{\dagger}$ Peter Hauff, ${ }^{\dagger}$ Thomas M. Zollner, ${ }^{\dagger}$ and Michael D. Menger*

From the Institute for Clinical and Experimental Surgery,* University of Saarland, Homburg/Saar; and Bayer Schering Pharma AG, ${ }^{\dagger}$ Global Therapeutic Research, Berlin, Germany

Endometriosis, the presence of endometrial tissue at ectopic sites, is a highly prevalent gynecological disease severely affecting a patient's quality of life. To analyze the mechanisms involved in the disease and to identify new molecular targets for effective therapies, small animal models are an important approach. Herein, we report the first use of high-resolution ultrasound imaging for the in vivo analysis of intraperitoneal endometriotic lesions in mice. This noninvasive technology allows for the repetitive quantitative analysis of growth, cyst development, and adhesion formation of endometriotic lesions with a low intra- and interobserver variability. Moreover, it enables one to easily differentiate between endometrial cysts and stroma. Accordingly, volume measurements of both endometrial cysts and stroma indicated that the initial establishment of endometriotic lesions is associated with enhanced cellular proliferation, followed by a phase of increased secretory activity of endometrial glands. Results of ultrasound analysis correlated well with measurements of lesion volumes by caliper and histology. Importantly, ultrasound imaging could be performed repetitively and noninvasively and reflected best the in vivo situation. The technique could further be demonstrated to successfully monitor the significant inhibition of growth of endometriotic lesions after specific estrogen receptor destabilizator treatment. Thus, high-resolution ultrasound imaging represents an important tool for future preclinical small animal studies, which address the pathophysiology of endometriosis and the development of new treatment strategies. ( $\mathrm{Am} \mathrm{J}$ Pathol 2010, 176:585-593; DOI: 10.2353/ajpath.2010.090617)

Endometriosis, the presence of endometrium-like tissue outside the uterine cavity, is one of the most frequent benign gynecological diseases. The most widely accepted theory for the development of endometriosis is retrograde menstruation, ie, reflux of endometrial fragments in the fallopian tubes during menstruation and their implantation and growth in the peritoneal cavity. ${ }^{1}$ However, retrograde menstruation is a physiological event, which occurs in up to $90 \%$ of women undergoing laparoscopy. ${ }^{2}$ Thus, other factors have been proposed to be involved in the pathogenesis of endometriosis, including immunological, inflammatory, genetic, environmental, and angiogenic determinants. ${ }^{3-6}$

For the development of effective strategies to treat endometriosis, genetically well defined rodent models are an important approach, because they allow standardized analysis of the mechanisms involved in the disease and the identification of new molecular targets for future medical therapies. In rodents, endometriotic lesions are typically induced by transplantation of uterine endometrium into the peritoneal cavity. ${ }^{7}$ However, this approach has the major disadvantage that repeated laparoscopies would be necessary to repetitively visualize engraftment and proliferation of individual lesions in vivo. Accordingly, data from these studies are normally based on histological, immunohistochemical, or gene expression analyses of isolated tissue samples at a single observation time point.

Accepted for publication October 27, 2009

Address reprint requests to Matthias W. Laschke, M.D., Institute for Clinical and Experimental Surgery, University of Saarland, D-66421 Homburg/Saar, Germany. E-mail: matthias.laschke@uniklinik-saarland.de. 
To overcome this problem, we herein introduce the technology of high-resolution ultrasound imaging for the noninvasive in vivo analysis of intraperitoneal endometriosis in mice. Our study demonstrates that high-resolution ultrasound is a reliable and easy method for the repetitive threedimensional assessment of growth, cyst development, and adhesion formation of endometriotic lesions. Moreover, it is an excellent tool to monitor the efficacy of novel medical therapies for the treatment of endometriosis.

\section{Materials and Methods}

\section{Animals}

For the study, 12- to 16-week-old female C57BL/6 mice with a body weight of 20 to $25 \mathrm{~g}$ were used. They were housed one per cage and had free access to tap water and standard pellet food (Altromin, Lage, Germany). Inbred animals were used to guarantee syngeneic endometrial tissue transplantation from donor into recipient animals and hence to prevent rejection of the transplanted tissue. Moreover, only those animals that were in an identical stage of the ovarian cycle, ie, estrus, were used for the experiments to exclude discrepancies in the growth behavior of developing endometriotic lesions between individual animals due to different sex hormone levels. Estrous cycling was evaluated by cytological analysis of vaginal lavage samples. For this purpose, $15 \mu \mathrm{l}$ of $0.9 \%$ saline was carefully pipetted into the vagina and subsequently transferred on a glass slide for examination under a phase-contrast microscope $(\mathrm{CH}-2$, Olympus, Hamburg, Germany). All experiments were approved by the local governmental animal care committee and were conducted in accordance with the German legislation on protection of animals and the National Institutes of Health Guide for the Care and Use of Laboratory Animals. ${ }^{8}$

\section{Model of Intraperitoneal Endometriosis}

Intraperitoneal endometriotic lesions were surgically induced as described previously with minor modifications. ${ }^{9-11}$ In brief, donor mice were anesthetized by i.p. injection of ketamine (75 mg/kg b.wt., Pharmacia GmbH, Erlangen, Germany) and 2\% xylazine (15 mg/kg b.wt., Rompun, Bayer, Leverkusen, Germany). Both uterine horns were removed and transferred into a Petri dish containing $37^{\circ} \mathrm{C}$ warm Dulbecco's modified Eagle medium (10\% fetal calf serum, $1 \mathrm{U} / \mathrm{ml}$ penicillin, and 0.1 $\mathrm{mg} / \mathrm{ml}$ streptomycin, PAA, Cölbe, Germany). The uterine horns were opened longitudinally with microscissors under a stereomicroscope (M651, Leica Microsystems $\mathrm{GmbH}$, Wetzlar, Germany), and tissue samples of comparable size were removed using a 2-mm dermal biopsy punch (Stiefel Laboratorium GmbH, Offenbach am Main, Germany). Then one tissue sample per quadrant was fixed with a 7-0 Prolene suture (Ethicon Products, Norderstedt, Germany) to the peritoneal wall of anesthetized recipient animals through a midline incision. Finally, the laparotomy was closed with a 6-0 Prolene muscle and skin suture. Subsequently, the endometriotic lesions were analyzed by high-resolution ultrasound imaging over a time period of 4 to 6 weeks.

\section{High-Resolution Ultrasound Image Acquisition}

Mice were anesthetized with $2 \%$ isoflurane in oxygen and fixed in the supine position on a heated stage with electrocardiogram electrodes and heart rate display (THM100, Indus Instruments, Houston, TX). After chemical depilation (Nair hair removal lotion, Church \& Dwight Canada Corp., Mississauga, ON, Canada) of the abdomen to prevent air trapped in the fur from interfering with ultrasound coupling into the animal, ultrasound coupling gel (Aquasonic 100, Parker, Fairfield, NJ) was applied to the skin.

Ultrasound imaging was performed with the Vevo 770 high-resolution imaging system (VisualSonics, Toronto, ON, Canada) and a real-time microvisualization 704 Scanhead (VisualSonics) with a center frequency of $40 \mathrm{MHz}$ and a focal depth of $6 \mathrm{~mm}$. For three-dimensional image acquisition, the ultrasound probe, driven by a linear motor, scanned the abdomen of the mouse. This procedure guaranteed that two-dimensional images were acquired at regular spatial intervals so they were parallel and uniformly spaced at $50-\mu \mathrm{m}$ steps. The predefined parallel geometry of the two-dimensional image planes enabled rapid threedimensional image reconstruction and display in a dynamic cube view format, as described previously. ${ }^{12}$ Scanning and three-dimensional image reconstruction of each endometriotic lesion took not more than $20 \mathrm{~s}$.

\section{Ultrasound Image Analysis}

Quantitative analysis of the ultrasound images was performed by means of a three-dimensional reconstruction and analysis software licensed to VisualSonics for distribution with the Vevo 770 high-resolution imaging system. The analysis included the determination of the overall volume (in $\mathrm{mm}^{3}$ ) of endometriotic lesions, their cysts, and their stroma by manual image segmentation. For this purpose, boundaries of endometriotic lesions and their cysts were manually outlined in parallel slices, separated by a defined step size in the three-dimensional ultrasound images. Based on the areas outlined, the volume of the endometriotic lesions was subsequently computed by the VisualSonics software.

To evaluate the impact of the step size chosen for manual image segmentation on the accuracy of computerassisted three-dimensional volume analysis, volume calculation was performed in one set of experiments using different step sizes ranging from 50 to $500 \mu \mathrm{m}$. Agreement between the data based on the step size of $50 \mu \mathrm{m}$ and larger step sizes was assessed by linear correlation analysis (Pearson's coefficient of correlation). Moreover, the volume deviation (in \%) of measurements with larger step sizes from those with a step size of $50 \mu \mathrm{m}$ was calculated and plotted against the results of the $50-\mu \mathrm{m}$ step size.

In another set of experiments, we analyzed the intraand interobserver variability of volume measurement by three independent examiners (C.K., J.R.-A. and M.W.L.), who were well experienced in ultrasound imaging of 
mice. For this purpose, the first examiner analyzed the volume of endometriotic lesions for two times at the interval of 1 month. The second and third examiners performed the volume measurement independently without knowledge of the previous results. Subsequently, the agreement between the data of the repeated measurements of the first examiner and the agreement between the data of the first, the second, and the third examiner were assessed by linear correlation analysis and calculation of deviations (in \%).

\section{Determination of Lesion Volume by Caliper Measurement and Histology}

At the end of the in vivo experiments the animals were anesthetized by i.p. injection of ketamine and $2 \%$ xylazine and carefully laparotomized under a stereomicroscope to measure the largest and the smallest diameter of the endometriotic lesions by means of a caliper. The volume of the endometriotic lesions was then calculated with the standard ellipsoid formula $D_{1}^{2} \times D_{2} \times \pi / 6$, where $D_{1}$ is defined as the smaller of the two diameters. ${ }^{13}$ Subsequently, the animals were sacrificed with an overdose of pentobarbital, and the endometriotic lesions were excised and processed for light microscopy. For this purpose, a tissue specimen of the abdominal wall of $\sim 12 \times 8 \mathrm{~mm}$ including the endometriotic lesion was excised. Before excision of the specimen, the laterolateral orientation was marked with a 6-0 Prolene suture at the cranial margin over the whole length of the specimen. Thus, the specimen could be embedded in known orientation of the lesion and could be cut into 5 - $\mu \mathrm{m}$-thick slices from caudal to cranial similarly as the ultrasound images were acquired. The slices were stained with H\&E according to standard procedures. Histological sections with a cutting interval of $50 \mu \mathrm{m}$ were examined under a light microscope (BX60, Olympus) and recorded on DVD by means of an AxioCam (Zeiss, Jena, Germany). Crosssectional areas of endometriotic lesions were measured by computer-assisted planimetry using Caplmage software (Zeintl, Heidelberg, Germany). To assess the volume of each lesion, trapezoidal bodies were calculated according to $V_{\mathrm{t}}=1 / 2 \times(A+B) \times d$, where $A$ and $B$ represent the neighboring section areas and $d$ represents the interslice distance. The sum of all trapezoidal bodies yielded the total lesion volume.

Agreement between the results of volume measurement based on ultrasound imaging, caliper measurement, and histological examination was assessed by linear correlation and Bland-Altman analysis. In addition, the deviation (in \%) of caliper and histological volume measurements from those of the ultrasound analysis was calculated and plotted against the computed volume of the ultrasound analysis.

\section{Experimental Protocol}

A total of 32 intraperitoneal endometriotic lesions were surgically induced in eight mice by transplanting four uterine tissue samples into each animal. Ultrasound image analysis of the volume of developing endometriotic lesions, their cysts, and their stroma was performed directly after tissue transplantation as well as at days $7,14,21$, and 28. At the end of the in vivo experiments, the animals were laparotomized again for volume measurement of endometriotic lesions by means of a caliper. Subsequently, the animals were sacrificed with an overdose of pentobarbital, and the endometriotic lesions were excised for histological examinations.

In an additional set of experiments, ultrasound imaging was used to monitor the effect of antiestrogenic therapy on endometriotic lesions. For this purpose, endometriotic lesions were treated with an established specific estrogen receptor destabilizer (SERD) (ZK191703, Bayer Schering Pharma, Berlin, Germany). A total of 72 intraperitoneal endometriotic lesions were surgically induced in 36 mice by transplanting two uterine tissue samples into each animal. The size of the lesions was analyzed 2 weeks after initial surgery by means of ultrasound imaging and macroscopic inspection during control laparotomy under a stereomicroscope followed by digital photography and measurement of lesion area (Axiovision, Zeiss). Subsequently, the animals were marked individually and randomly grouped into 18 vehicle controls and 18 SERD-treated mice. The animals were treated daily from day 14 onward to day 42 with $1 \mathrm{mg} / \mathrm{kg} /$ day SERD dissolved in ethanol/peanut oil (5:95) or with vehicle only. After 4 weeks of treatment, the endometriotic lesions were again investigated by ultrasound imaging and macroscopic evaluation.

\section{Statistics}

To test for time effects, analysis of variance for repeated measures was applied. This was followed by StudentNewman-Keuls pairwise comparisons, including correction of the $\alpha$ error to compensate for multiple comparisons (SigmaStat, Jandel Corporation, San Rafael, CA). For Student-Newman-Keuls pairwise comparisons, sample means are ordered from the smallest to the largest. The largest difference, which involves means that are $r=$ $p$ steps apart, is tested first at the $\alpha$ level of significance. If significant, means that are $r=p-1$ steps apart are subsequently tested at $\alpha$ level of significance and so on. Differences between SERD- and vehicle-treated animals were calculated by the Mann-Whitney rank-sum test. In addition, linear correlation (Pearson's coefficient of correlation) and Bland-Altman analysis were performed to evaluate correspondence of different volume measurement techniques. To create a Bland-Altman plot, the mean for volumes of endometriotic lesions measured by ultrasound and caliper or histological examination was plotted on the horizontal axis, and the differences between the two types of measurement were plotted on the vertical axis. ${ }^{14,15}$ Values are expressed as means \pm SEM for parametric distribution or box plots for nonparametric distribution. Statistical significance was accepted for a value of $P<0.05$. 


\section{Results}

\section{Ultrasound Imaging of Endometriotic Lesion Formation}

Suturing of uterine tissue samples to the lateral abdominal wall provided optimal conditions for repetitive ultrasound imaging of developing endometriotic lesions (Figure $1, A-C)$. Directly after transplantation, they presented as homogeneous tissue masses fixed to the peritoneum (Figure 1D). Because their position was not affected by respiratory movements or peristalsis of the intestine, they could easily be distinguished from the adjacent intraabdominal organs. During the following observation period, the development of anechoic cysts was detected within the grafts, which is a typical sign for the establishment of endometriotic lesions ${ }^{16}$ (Figure 1, E-H). The localization and extension of these cysts could be nicely visualized in three-dimensional reconstructions of the lesions using VisualSonics software (Figure 1, J and K). Moreover, high-resolution ultrasound imaging allowed noninvasive visualization of adhesion formation around the lesions for the first time (Figure 1, L and M). Histological examination of tissue samples at the end of the in vivo experiments, ie, day 28 , confirmed our observations during ultrasound imaging. The uterine grafts had developed to typical endometriotic lesions, which were characterized by cyst-like dilated endometrial glands surrounded by a well vascularized endometrial stroma (Figure 1I).

Quantitative analysis of ultrasound images demonstrated that the developing endometriotic lesions grew during the 4-week observation period, as indicated by a significantly increased lesion volume of $2.0 \mathrm{~mm}^{3}$ at day 28 when compared with that of day $0\left(0.9 \mathrm{~mm}^{3}\right)$ (Figure $1 \mathrm{~N})$. However, more detailed analysis of stroma and cyst
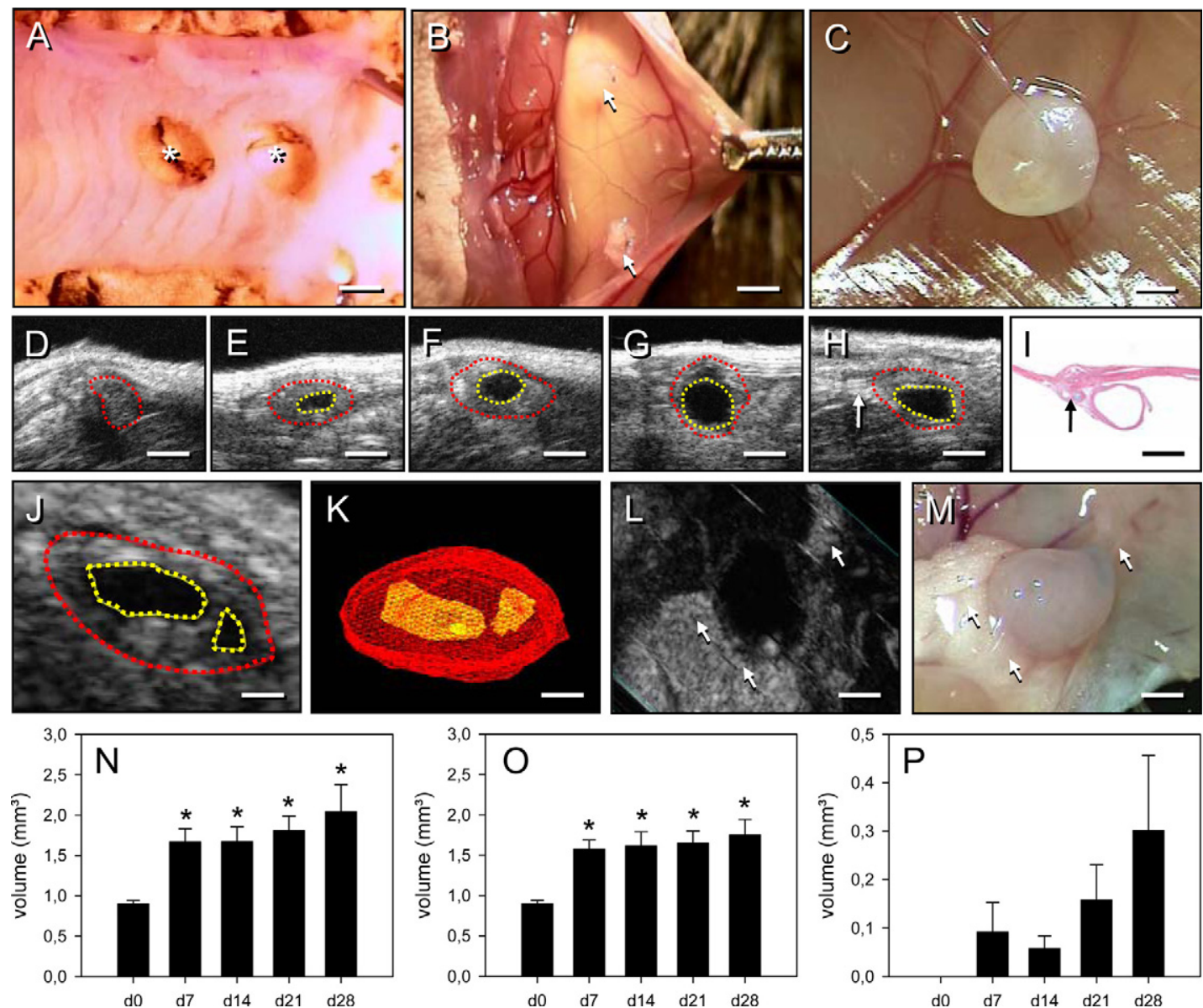

Figure 1. A-C: Surgical induction of intraperitoneal endometriosis. A: For this purpose, uterine tissue samples of comparable size are removed from a longitudinally opened uterine horn of a donor animal by means of a biopsy punch (asterisks). B: The tissue samples are then sutured to the peritoneal wall of a recipient animal (arrows). C: After 4 weeks, the fixed uterine tissue samples have developed to vascularized endometriotic lesions with fluid-filled cysts. Scale bars: $1.3 \mathrm{~mm}(\mathbf{A}) ; 1.9 \mathrm{~mm}(\mathbf{B}) ; 0.2 \mathrm{~mm}(\mathbf{C})$. D-H: High-resolution ultrasound imaging of a developing endometriotic lesion (borders marked by red broken line) directly $(\mathbf{D})$ as well as $7(\mathbf{E}), 14(\mathbf{F}), 21(\mathbf{G})$, and 28 days $(\mathbf{H})$ after fixation of a uterine tissue sample to the abdominal wall. The formation of anechoic cysts $(\mathbf{E}-\mathbf{H}$, borders marked by yellow broken line) can easily be visualized with this noninvasive technique. H and I: Comparison of a cross-sectional plane of the ultrasound image $(\mathbf{H})$ with a H\&E-stained histology slide at day 28 after transplantation (I). Histological analysis shows the typical histomorphology of an endometriotic lesion, consisting of a cyst-like dilated endometrial gland surrounded by a well vascularized endometrial stroma (I). Note that a granuloma (arrow) has developed around the suture for initial fixation of the uterine tissue to the abdominal wall. The suture can be differentiated from the endometriotic lesion by ultrasound imaging due its echogenic pattern (H, arrow). Scale bars: $700 \mu \mathrm{m}(\mathbf{D}-\mathbf{I})$. $\mathbf{J}$ and $\mathbf{K}$ : Three-dimensional reconstruction of an endometriotic lesion (K, red polygon) with two cysts (K, yellow polygon) based on manual image segmentation of two-dimensional ultrasound images ( $\mathbf{J}$ ). Scale bars: $370 \mu \mathrm{m}$. $\mathbf{L}$ and $\mathbf{M}$ : High-resolution ultrasound image of adhesions ( $\mathbf{L}$, arrows) around an endometriotic lesion and the corresponding stereomicroscopic appearance of the adhesions ( $\mathbf{M}$, arrows) after laparotomy of the animal. Scale bars: $900 \mu \mathrm{m}$. N-P: Quantitative analysis of the overall volume (in $\left.\mathrm{mm}^{3}\right)$ of endometriotic lesions $(\mathbf{N})$, their stroma $(\mathbf{O})$, and their cysts $(\mathbf{P})$, as assessed by manual image segmentation and computer-assisted three-dimensional volume calculation. Means \pm SEM. ${ }^{*} P<0.05$ versus day 0 . 

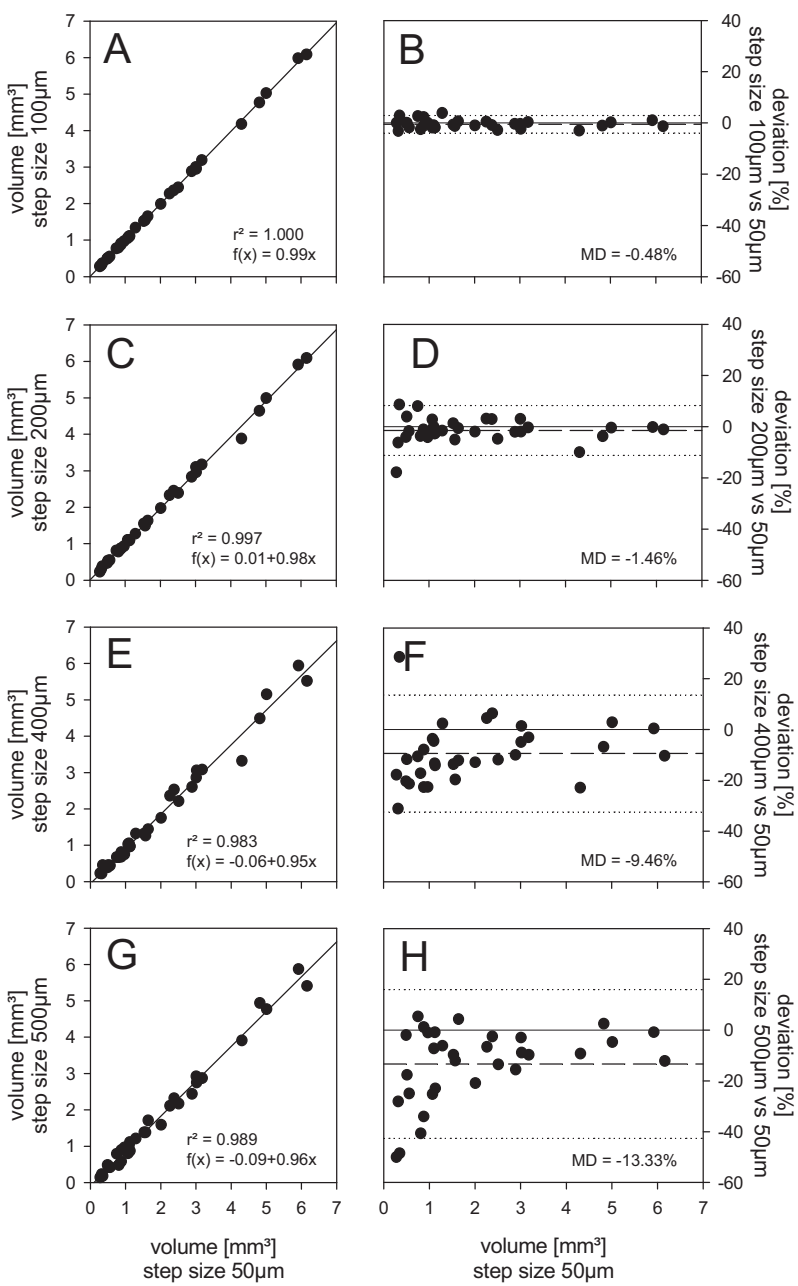

Figure 2. Impact of the step size chosen for manual image segmentation on the accuracy of computer-assisted three-dimensional volume calculation, as assessed by linear correlation analysis $(\mathbf{A}, \mathbf{C}, \mathbf{E}$, and $\mathbf{G}$ ) and calculation of deviations (in \%) $(\mathbf{B}, \mathbf{D}, \mathbf{F}$, and $\mathbf{H})$. Volume calculation of endometriotic lesions $(n=32)$ was performed using step sizes ranging from 50 to $500 \mu \mathrm{m}$. The results were plotted against the volume measurement based on the $50-\mu \mathrm{m}$ step size. Volume deviation (in \%) of measurements with larger step sizes from those with a step size of $50 \mu \mathrm{m}$ was calculated and plotted against the results of the $50 \mu \mathrm{m}$ step size. MD, mean deviation; dashed line, mean deviation; dotted lines, double SD.

volume revealed that the tissue mass of the lesions only increased during the first 7 days after transplantation of uterine samples into the abdominal cavity (Figure 1, 0 and $\mathrm{P}$ ). In the further time course of the experiment, growth of endometriotic lesions was caused mainly by an increase in cyst volumes.

\section{Correlation of Volume Measurements Based on Different Step Sizes}

Linear regression analysis of volume measurements based on a step size of $50 \mu \mathrm{m}$ with that of larger step sizes showed a high correlation (Figure 2, A, C, E, and $G)$. However, the mean deviation of volume measurements progressively increased from a mean deviation of $-0.48 \%$ for a step size of $100 \mu \mathrm{m}$ to $-13.33 \%$ for a step size of $500 \mu \mathrm{m}$ (Figure 2, B, D, F, and H). These data
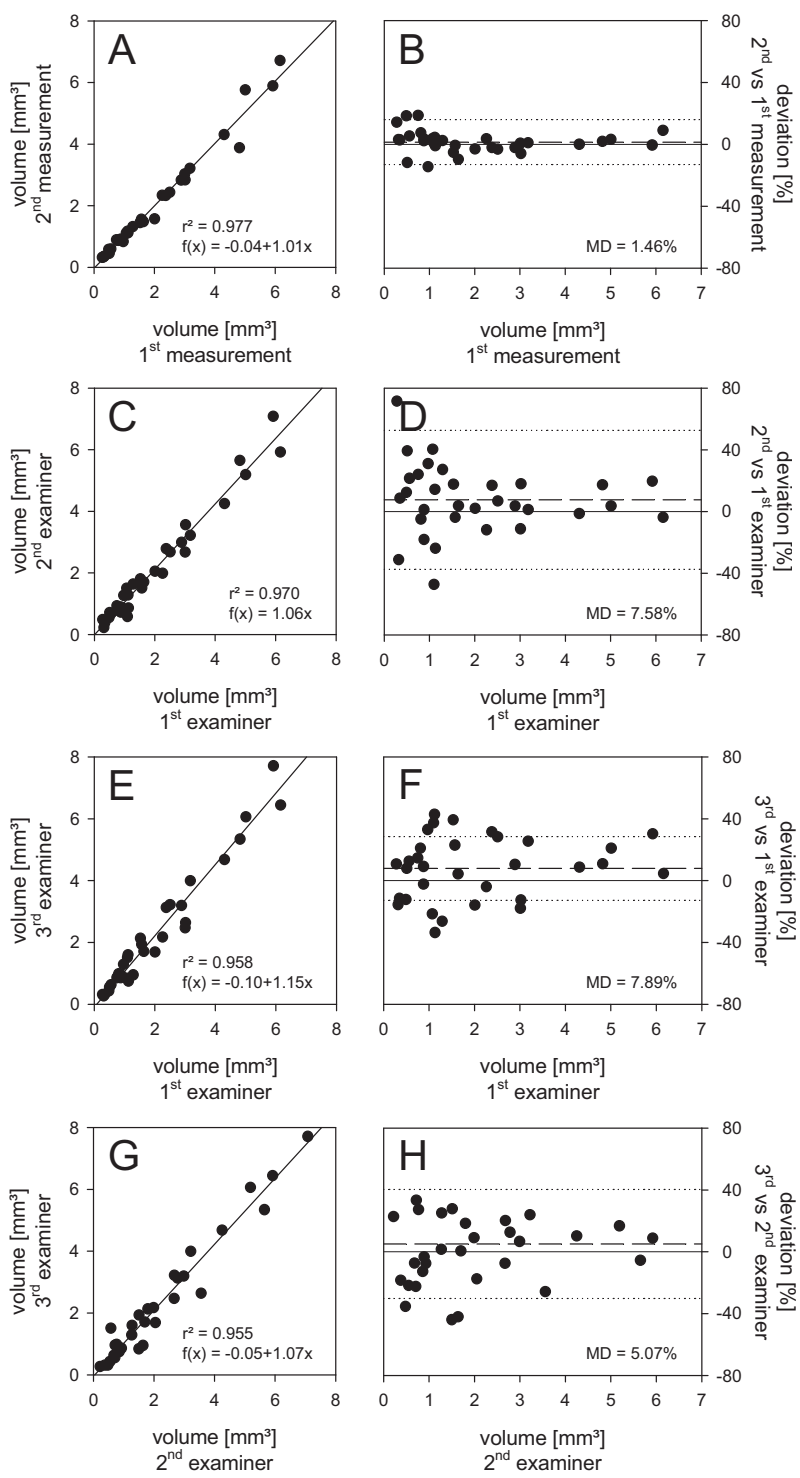

Figure 3. Intraobserver variability $(\mathbf{A}$ and $\mathbf{B})$ and interobserver variability $(\mathbf{C}-\mathbf{H})$ of volume measurements of endometriotic lesions $(n=32)$ by highresolution ultrasound imaging, as assessed by linear correlation analysis (A $\mathbf{C}, \mathbf{E}$, and $\mathbf{G}$ ) and calculation of deviations (in \%) (B, D, F, and $\mathbf{H})$. MD, mean deviation; dashed line, mean deviation; dotted lines, double SD.

demonstrate that the volume of endometriotic lesions is increasingly underestimated, when larger step sizes are used for three-dimensional volume calculation. Closer examination of the distribution pattern of endometriotic lesions of different sizes revealed that this deviation was most pronounced for small lesions with a volume $<1 \mathrm{~mm}^{3}$.

\section{Intra- and Interobserver Variability}

The linear correlation between the two sets of volume measurements performed by the first examiner (intraobserver variability) was high with $r^{2}=0.977$ (Figure 3A). The mean deviation of individual measurements was $1.46 \%$ (Figure 3B). In addition, comparison between the volume measurements of the first, the second, and the third examiner (interobserver variability) also revealed a 

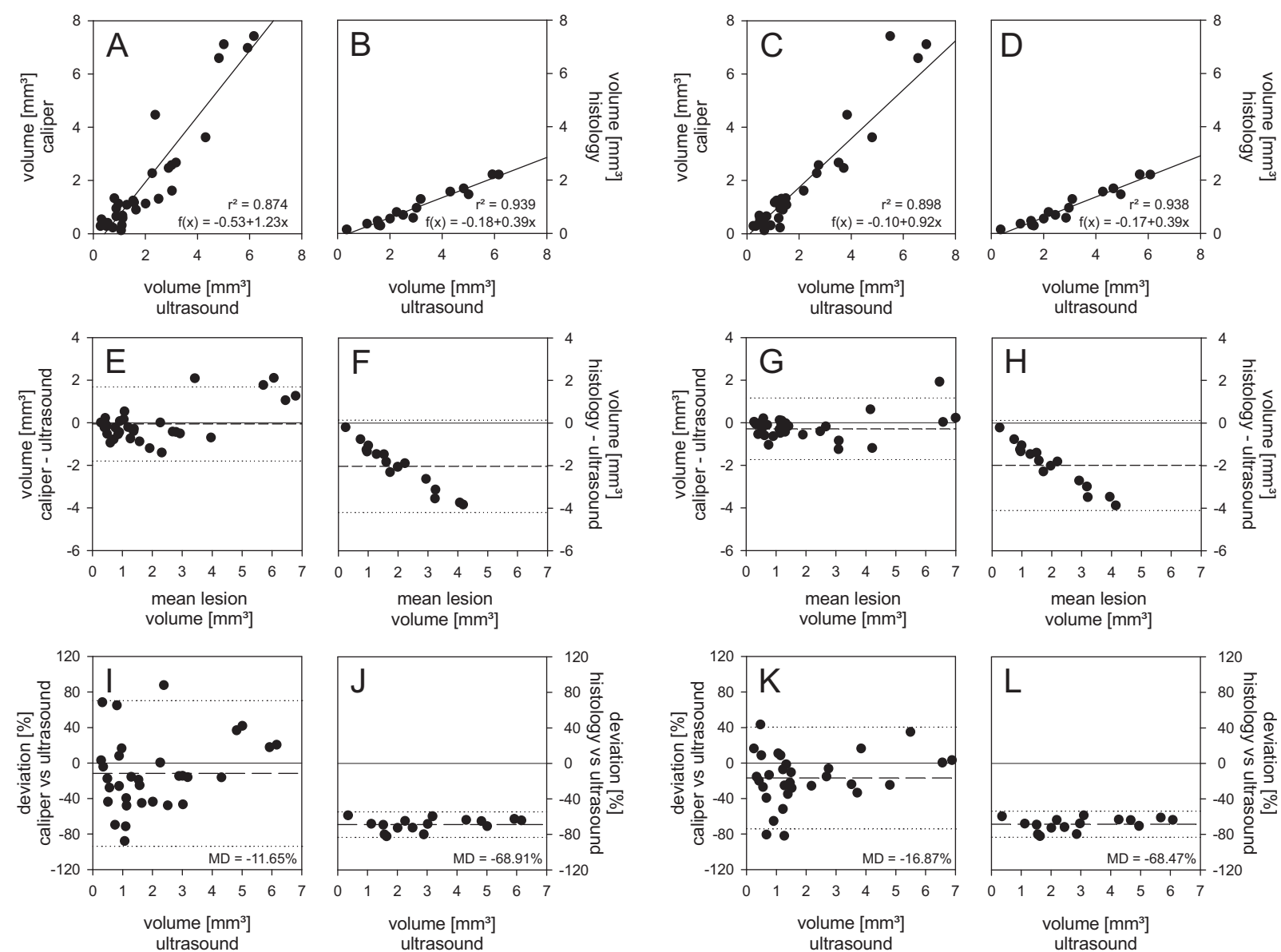

Figure 4. Correspondence of volume measurement of endometriotic lesions by high-resolution ultrasound imaging with volume measurement by caliper (A, E, and $\mathbf{I} ; n=32$ ) and histological examination ( $\mathbf{B}, \mathbf{F}$, and $\mathbf{J} ; n=16)$, as assessed by linear correlation analysis (A and $\mathbf{B})$, Bland-Altman analysis (E and $\mathbf{F})$, and calculation of deviations (in \%) (I and $\mathbf{J}$ ). To create a Bland-Altman plot, the mean for volumes of endometriotic lesions measured by ultrasound and caliper or histological examination was plotted on the horizontal axis and the differences between the two types of measurement were plotted on the vertical axis. In $\mathbf{C}$, $\mathbf{D}, \mathbf{G}, \mathbf{H}, \mathbf{K}$, and $\mathbf{L}$ corresponding analyses were performed. However, lesion volumes determined by ultrasound were calculated according to the caliper measurement (assessment of largest and smallest diameter) and histological examination (trapezoidal interpolation), allowing us to assess the influences of different methods for data acquisition by comparing $\mathbf{A}, \mathbf{B}, \mathbf{E}, \mathbf{F}, \mathbf{I}$, and $\mathbf{J}$ with $\mathbf{C}, \mathbf{D}, \mathbf{G}, \mathbf{H}, \mathbf{K}$, and $\mathbf{L}$. MD, mean deviation; dashed line, mean deviation; dotted lines, double SD.

high correlation with $r^{2}=0.970$ (first versus second examiner, Figure 3C), $r^{2}=0.958$ (first versus third examiner, Figure 3E), and $r^{2}=0.955$ (second versus third examiner, Figure $3 \mathrm{G}$ ). The deviations of the measurements obtained by the second and the third examiner from those of the first examiner were found to be dispersed around a mean deviation of 7.58 and $7.89 \%$ with no clear trend toward over- or underestimation by either one of these examiners (Figure 3, D and F). Comparable results were found for the deviation of the measurements obtained by the third examiner from those of the second examiner (Figure $3 \mathrm{H}$ ). Notably, the deviation was most pronounced for small endometriotic lesions with a volume $<1.5 \mathrm{~mm}^{3}$.

\section{Correlation between Ultrasound Analysis and Volume Measurement by Caliper and Histology}

For the comparison between ultrasound-based three-dimensional volume calculation and volume calculation by caliper measurement the coefficient of correlation was found to be $r^{2}=0.874$ (Figure $4 \mathrm{~A}$ ), which still has to be considered as a good correlation between the two methods. There was no clear trend toward an over- or underestimation of the volume of endometriotic lesions by either one of these methods. Moreover, almost all values were located within the double SD agreement limits of the Bland-Altman plot (Figure 4E). However, for individual endometriotic lesions large deviations of caliper measurements from those of ultrasound imaging of up to $87 \%$ were found (Figure 4l).

The coefficient of correlation between ultrasound and histological analysis was $r^{2}=0.939$ (Figure 4B). However, in contrast to the volume measurement by means of caliper, histologically determined volumes of endometriotic lesions were basically underestimated compared with the ultrasound measurement. In fact, Bland-Altman analysis showed that the difference between histological and ultrasound volumes arithmetically increased with larger lesion volumes (Figure 4F). Accordingly, volume deviations of individual lesions were found to be closely distributed around a mean deviation of $-68.91 \%$ (Figure 4J). 

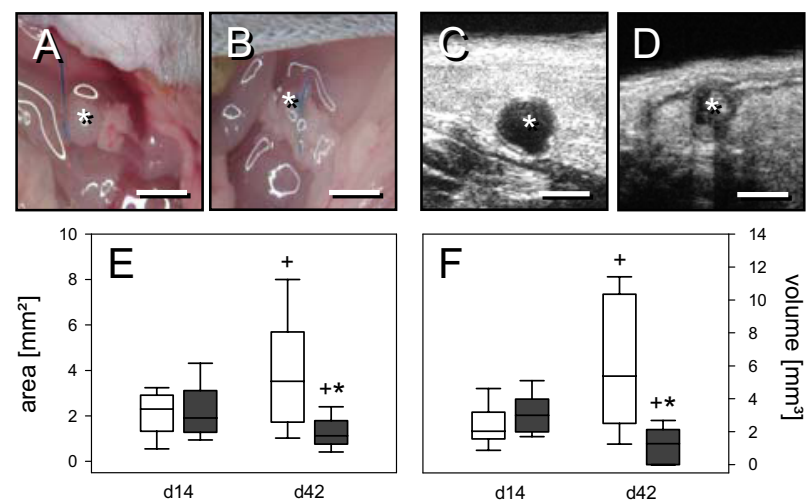

Figure 5. A-D: Macroscopic evaluation ( $\mathbf{A}$ and $\mathbf{B}$ ) and ultrasound imaging (C and D) of established endometriotic lesions (asterisks) at day 42 after suturing uterine tissue samples to the peritoneal wall of animals, which were treated daily from day 14 onward to day 42 with a SERD (B and $\mathbf{D}$ ) or with vehicle (A and $\mathbf{C})$. Note that SERD treatment reduces the lesion size. Scale bars: $2.5 \mathrm{~mm}$ (A and $\mathbf{B}) ; 2.7 \mathrm{~mm}(\mathbf{C}$ and $\mathbf{D})$. E and $\mathbf{F}$ : Quantitative analysis of the area (in $\mathrm{mm}^{2}$ ) and the overall volume (in $\mathrm{mm}^{3}$ ) of endometriotic lesions in SERD-treated (gray box plots) and vehicle-treated animals (white box plots) at day 14 and day 42, as assessed by macroscopic evaluation (E) and ultrasound imaging using computer-assisted three-dimensional volume calculation $(\mathbf{F}) .{ }^{*} P<0.05$ versus vehicle; ${ }^{* * *} P<0.05$ versus day 14 .

\section{Ultrasound Imaging in a Therapeutic Setting}

It is well known that endometriosis is an estrogen-dependent disease. Accordingly, we treated endometriotic lesions with a SERD. As expected, we could show by invasive macroscopic evaluation of established endometriotic lesions that SERD treatment resulted in a significantly reduced lesion area at day 42 compared with controls (Figure 5, A, B, and E). Importantly, ultrasoundbased three-dimensional volume calculation of the lesions could confirm these results (Figure 5, C, D, and F), indicating that high-resolution ultrasound imaging is a reliable tool to monitor noninvasively the efficacy of medical therapies for endometriosis.

\section{Discussion}

In the present study we introduce high-resolution ultrasound imaging for the in vivo analysis of endometriotic lesions in mice. Using scanheads with a high frequency of $40 \mathrm{MHz}$ to permit a resolution of $30 \mu \mathrm{m}$ at a focal depth of $6 \mathrm{~mm}$, this technology allows for the repetitive and noninvasive determination of growth of endometriotic lesions with good soft tissue contrast and without the need for i.v. contrast agents. The animals tolerated the repeated imaging sessions well, which may be due to the use of volatile rather than injection anesthetics as well as the noninvasive nature of ultrasound exposure. Accordingly, ultrasound imaging may significantly contribute to limiting the statistical variability of preclinical endometriosis studies by monitoring individual endometriotic lesions over time. This will reduce the number of animals required for a study and will maximize the amount of data obtained from each animal. The small intra- and interobserver variability of lesion volume measurements further supports the validity of the analysis based on this technology.
For experimental studies, endometriotic lesions are usually induced by transplanting endometrium to ectopic sites, because the development of spontaneous endometriosis is dependent on menstruation and thus restricted to humans and subhuman primates. ${ }^{7}$ In the past, several studies have used the chorioallantoic membrane of the chicken embryo ${ }^{17-19}$ or the dorsal skinfold chamber of the Syrian golden hamster ${ }^{20-22}$ as the host site for endometrial tissue transplantation. Both models bear the major advantage that endometriotic lesions can be analyzed repetitively and noninvasively. However, they are restricted in use to a time period of a few days to weeks. Moreover, they may not exactly reflect the physiological profile of cytokines and growth factors of the peritoneal fluid and the intraabdominal immune system.

Alternatively, endometrium may be transplanted directly into the peritoneal cavity of rodents. This, however, involves the opening of the abdomen, which may be associated with surgically induced inflammation and wound healing, resulting in the nonphysiological composition of peritoneal fluid and hence altered development of intraperitoneal endometriotic lesions. Repeated laparotomies to study development of endometriotic lesions in one individual over time may further enhance these inflammatory responses. Accordingly, in many studies using this approach cohorts of animals are sacrificed for measurements of endometriotic lesions at different time points. In fact, repeated laparoscopies would be necessary to visualize engraftment and proliferation of individual lesions in one animal over time.

To overcome this problem, Becker et $\mathrm{al}^{9}$ recently established a novel model of peritoneal endometriosis, which allows for the noninvasive monitoring of endometriotic lesions using the technique of bioluminescence. However, for this purpose endometrium of luciferaseexpressing transgenic mice, which illuminates selectively in wild-type mice after systemic injection of luciferin has to be used. In contrast, the technique of ultrasound imaging introduced herein is not dependent on transgenic mouse models and provides a considerably higher spatial resolution than the bioluminescence technology.

In our rodent model of intraperitoneal endometriosis, uterine tissue samples were sutured to the lateral abdominal wall, providing optimal conditions for repetitive ultrasound imaging of developing endometriotic lesions. Using this approach one should be aware that every implanted suture biomaterial induces a more or less strong foreign body reaction that may affect the development of the lesions. Alternatively, endometriosis may be induced by intraperitoneal injections of suspensions containing endometrial tissue fragments. ${ }^{23,24}$ However, in this model it is quite difficult and time-consuming to detect and analyze the randomly distributed endometriotic lesions in the peritoneal cavity by ultrasound imaging. Moreover, previous studies demonstrated that results obtained from sutured endometriotic lesions correlated well with immunohistochemical results from endometriotic lesions that were not attached surgically to the peritoneal wall. ${ }^{9}$ Therefore, we suggest that future ultrasound studies should be performed in the surgical endometriosis model. 
Histologically, endometriotic lesions are characterized by cyst-like dilated endometrial glands, which are surrounded by a vascularized endometrial stroma. ${ }^{25}$ Interestingly, we could easily differentiate between these compartments by the in vivo ultrasound analysis of the lesions. Accordingly, we were able to quantify the growth of both endometrial cysts and stroma over the 4-week observation period. By this, we found that the tissue mass of the lesions only increased during the first 7 days, whereas in the further time course lesion growth was mainly caused by the formation of cysts. This observation indicates that, in particular, the initial establishment of endometriotic lesions is associated with enhanced cellular proliferation, followed by a phase of increased secretory activity of the endometrial glands. Hence, it may not be sufficient to measure only the overall size of endometriotic lesions, when the efficacy of new treatment strategies for the therapy of endometriosis is tested. In fact, by means of high-resolution ultrasound imaging it is now possible to analyze in more detail the distinct effects of various therapies on cell proliferation and on secretory activity of endometriotic lesions.

Quantitative analysis of the ultrasound images was performed by manual image segmentation. The accuracy of this method is critically dependent on the step size of the two-dimensional ultrasound images analyzed for three-dimensional volume calculation and lesion detection. According to the Nyquist-Shannon sampling theorem, a step size of $50 \mu \mathrm{m}$ is capable of detecting an object with a diameter of $\sim 100 \mu \mathrm{m}$. In the present study, the smallest endometriotic lesion to be reproduced was $\sim 800 \mu \mathrm{m}$ in diameter (lesion with a size of $0.27 \mathrm{~mm}^{3}$ as determined with the $50-\mu \mathrm{m}$ step size analysis). This finding indicates that a step size equal to $400 \mu \mathrm{m}$ or less should be applied to fulfill the requirements of the Nyquist-Shannon sampling theorem for object detection, which is nicely confirmed by the calculations of deviations of volume measurements, indicating in small lesions $<1 \mathrm{~mm}^{3}$ an increase in deviation (underestimation) of lesion volumes with 400- $\mu \mathrm{m}$ and, in particular, 500- $\mu \mathrm{m}$ step size analysis. This increase in deviation of volume measurements in lesions $<1 \mathrm{~mm}^{3}$ may not necessarily be due to the fact that the lesions could not be detected according to the Nyquist-Shannon sampling theorem (sampling interval not less than half of the size of the object), because the diameter of a $0.5-\mathrm{mm}^{3}$ lesion is $\sim 1000 \mu \mathrm{m}$ and, thus, detectable with a step size of 400 or $500 \mu \mathrm{m}$. The increase in deviation of volume measurements in small lesions is rather due to the fact that with a step size of 400 or $500 \mu \mathrm{m}$ the volume of the lesions is less exactly determined. Indeed, if the lesion diameter is not exactly a multiple of the step size used, manual image segmentation analysis starting at one end of the lesion causes the distant end of the lesion to be lost for volume measurement. In relation to the overall volume of a lesion, the volume of this lost end is relatively high in smaller lesions compared with that in larger lesions. Thus, a large step size on small volumes is not precise enough, whereas a large step size on large volumes may be appropriate.
Because the determination of lesion size by means of a caliper is a common procedure in experimental endometriosis studies, ${ }^{10,11}$ we correlated this method with our ultrasound analysis. Interestingly, we show that there is good correlation between both methods. However, for individual endometriotic lesions large deviations of caliper measurements from those of ultrasound imaging could be found. Therefore, we suggest that caliper measurement should be used to confirm results obtained from ultrasound imaging rather than for the exclusive generation of reliable data on lesion growth. In contrast, volumes of endometriotic lesions measured by histological analysis were basically underestimated compared with the ultrasound measurement. This finding is probably due to the fact that the volume of endometriotic lesions drastically decreases during the processing of tissue samples for histological analysis compared with the in vivo situation, which is much better reflected by caliper measurement and ultrasound imaging. Nonetheless, histological examination is still an essential approach, when one is analyzing cellular processes within endometriotic lesions, such as angiogenesis, cell proliferation, apoptotic cell death, or recruitment of inflammatory cells. In fact, histological analyses may explain data on growth or regression of endometriotic lesions, which have been assessed by ultrasound imaging.

In line with the fact that endometriosis is an estrogendependent disease, we could further show in our study by invasive macroscopic evaluation of established endometriotic lesions that SERD treatment resulted in a significant reduction of the lesion size over time. Importantly, these results could be reproduced by noninvasive ultrasound-based three-dimensional volume calculation of the lesions. Therefore, we suggest that high-resolution ultrasound imaging is also a valid tool to monitor the efficacy of medical therapies for endometriosis.

In summary, the present study demonstrates that highresolution ultrasound imaging is a reliable and easy method for the repetitive analysis of intraperitoneal endometriosis in small animal models. This noninvasive technology provides images with a high spatial resolution that allow for the first time detailed qualitative and quantitative analysis of growth, cyst development, and adhesion formation of endometriotic lesions. Accordingly, high-resolution ultrasound imaging represents a versatile tool in preclinical endometriosis research, not only to gain new insights into the pathophysiology of the disease but also to develop future treatment strategies.

\section{Acknowledgments}

We thank Michaela Adams, Janine Becker, Christine Morgenroth, Nicole Müller, and Julia Parakenings for excellent technical assistance.

\section{References}

1. Sampson JA: Peritoneal endometriosis due to menstrual dissemination of endometrial tissues into the peritoneal cavity. Am J Obstet Gynecol 1927, 14:422-469 
2. Halme J, Hammond MG, Hulka JF, Raj SG, Talbert LM: Retrograde menstruation in healthy women and in patients with endometriosis. Obstet Gynecol 1984, 64:151-154

3. Zondervan KT, Cardon LR, Kennedy SH: The genetic basis of endometriosis. Curr Opin Obstet Gynecol 2001, 13:309-314

4. Berkkanoglu M, Arici A: Immunology and endometriosis. Am J Reprod Immunol 2003, 50:48-59

5. Laschke MW, Menger MD: In vitro and in vivo approaches to study angiogenesis in the pathophysiology and therapy of endometriosis. Hum Reprod Update 2007, 13:331-342

6. Anger DL, Foster WG: The link between environmental toxicant exposure and endometriosis. Front Biosci 2008, 13:1578-1593

7. Grümmer R: Animal models in endometriosis research. Hum Reprod Update 2006, 12:641-649

8. National Research Council: Guide for the Care and Use of Laboratory Animals. DHHS Publication No. (NIH) 85-23 (Rev.). Washington, DC, Department of Health and Human Services, 1985

9. Becker CM, Wright RD, Satchi-Fainaro R, Funakoshi T, Folkman J, Kung AL, D'Amato RJ: A novel noninvasive model of endometriosis for monitoring the efficacy of antiangiogenic therapy. Am J Pathol 2006, 168:2074-2084

10. Becker CM, Rohwer N, Funakoshi T, Cramer T, Bernhardt W, Birsner A, Folkman J, D'Amato RJ: 2-Methoxyestradiol inhibits hypoxia-inducible factor- $1 \alpha$ and suppresses growth of lesions in a mouse model of endometriosis. Am J Pathol 2008, 172:534-544

11. Fainaru O, Adini A, Benny O, Adini I, Short S, Bazinet L, Nakai K, Pravda E, Hornstein MD, D'Amato RJ, Folkman J: Dendritic cells support angiogenesis and promote lesion growth in a murine model of endometriosis. FASEB J 2008, 22:522-529

12. Fenster A, Downey DB, Cardinal HN: Three-dimensional ultrasound imaging. Phys Med Biol 2001, 46:R67-R99

13. Tomayko MM, Reynolds CP: Determination of subcutaneous tumor size in athymic (nude) mice. Cancer Chemother Pharmacol 1989, $24: 148-154$

14. Bland JM, Altman DG: Statistical methods for assessing agreement between two methods of clinical measurement. Lancet 1986, 1:307-310

15. Bland JM, Altman DG: Applying the right statistics: analyses of measurement studies. Ultrasound Obstet Gynecol 2003, 22:85-93
16. Athey PA, Diment DD: The spectrum of sonographic findings in endometriomas. J Ultrasound Med 1989, 8:487-491

17. Maas JW, Groothuis PG, Dunselman GA, de Goeij AF, StruijkerBoudier HA, Evers JL: Development of endometriosis-like lesions after transplantation of human endometrial fragments onto the chick embryo chorioallantoic membrane. Hum Reprod 2001, 16:627-631

18. Hull ML, Charnock-Jones DS, Chan CL, Bruner-Tran KL, Osteen KG, Tom BD, Fan TP, Smith SK: Antiangiogenic agents are effective inhibitors of endometriosis. J Clin Endocrinol Metab 2003, 88:2889-2899

19. Nap AW, Groothuis PG, Demir AY, Maas JW, Dunselman GA, de Goeij AF, Evers JL: Tissue integrity is essential for ectopic implantation of human endometrium in the chicken chorioallantoic membrane. Hum Reprod 2003, 18:30-34

20. Laschke MW, Elitzsch A, Scheuer C, Holstein JH, Vollmar B, Menger MD: Rapamycin induces regression of endometriotic lesions by inhibiting neovascularization and cell proliferation. $\mathrm{Br} J$ Pharmacol 2006, 149:137-144

21. Laschke MW, Elitzsch A, Scheuer C, Vollmar B, Menger MD: Selective cyclo-oxygenase-2 inhibition induces regression of autologous endometrial grafts by down-regulation of vascular endothelial growth factor-mediated angiogenesis and stimulation of caspase-3-dependent apoptosis. Fertil Steril 2007, 87:163-171

22. Laschke MW, Schwender C, Scheuer C, Vollmar B, Menger MD Epigallocatechin-3-gallate inhibits estrogen-induced activation of endometrial cells in vitro and causes regression of endometriotic lesions in vivo. Hum Reprod 2008, 23:2308-2018

23. Hirota $Y$, Tranguch S, Daikoku T, Hasegawa A, Osuga Y, Taketani Y, Dey SK: Deficiency of immunophilin FKBP52 promotes endometriosis. Am J Pathol 2008, 173:1747-1757

24. Styer AK, Sullivan BT, Puder M, Arsenault D, Petrozza JC, Serikawa T, Chang S, Hasan T, Gonzalez RR, Rueda BR: Ablation of leptin signaling disrupts the establishment, development, and maintenance of endometriosis-like lesions in a murine model. Endocrinology 2008, 149:506-514

25. Albee RB Jr, Sinervo K, Fisher DT: Laparoscopic excision of lesions suggestive of endometriosis or otherwise atypical in appearance: relationship between visual findings and final histologic diagnosis. J Minim Invasive Gynecol 2008, 15:32-37 